\title{
An altering distance function in fuzzy metric fixed point theorems
}

\author{
Nopparat Wairojjana ${ }^{1 *}$, Tatjana Došenović 2 , Dušan Rakić ${ }^{2}$ Dhananjay Gopal ${ }^{3}$ and Poom Kumam ${ }^{4 *}$
}

\author{
"Correspondence: \\ noparatw.ipad@gmail.com; \\ poom.kum@kmutt.ac.th \\ ${ }^{1}$ Faculty of Science and Technology, \\ Valaya Alongkorn Rajabhat \\ University under the Royal \\ Patronage, 1 Moo 20 Phaholyothin \\ Rd., Klong Neung, Klong Luang, \\ Pathumthani, 13180, Thailand \\ ${ }^{4}$ Department of Mathematics, \\ Faculty of Science, King Mongkut's \\ University of Technology Thonburi \\ (KMUTT), 126 Pracha Uthit Rd., Bang \\ Mod, Thung Khru, Bangkok, 10140, \\ Thailand \\ Full list of author information is \\ available at the end of the article
}

\begin{abstract}
The aim of this paper is to improve conditions proposed in recently published fixed point results for complete and compact fuzzy metric spaces (Ćirić in Chaos Solitons Fractals 42:146-154, 2009; Shen et al. in Appl. Math. Lett. 25:138-141, 2012). For this purpose, the altering distance functions are used. Moreover, in some of the results presented the class of $t$-norms is extended by using the theory of countable extensions of $t$-norms. The mentioned generalizations are illustrated by examples.
\end{abstract}

MSC: Primary 54H25; secondary $47 \mathrm{H} 10$

Keywords: fixed point; Cauchy sequence; $t$-norm; altering distance; fuzzy metric space

\section{Introduction and preliminaries}

In the fixed point theory in metric space an important place occupies the Banach contraction principle [1]. The mentioned theorem is generalized in metric spaces as well as in its various generalizations. In particular, the Banach contraction principle is observed in fuzzy metric spaces. There are several definitions of the fuzzy metric space [2-4]. George and Veeramani introduced the notion of a fuzzy metric space based on the theory of fuzzy sets, earlier introduced by Zadeh [5], and they obtained a Hausdorff topology for this type of fuzzy metric spaces [2,3]. For more information as regards the fuzzy metric and probabilistic metric spaces and fixed point theory in these spaces, we recommend [6-15].

The theory of fuzzy sets has broad applications in a variety of sciences including medicine, science of neural networks, control theory, engineering sciences, soil sciences, modeling some physical phenomena, etc. (see for example [16-20]). Moreover, there are many possibilities for improvement in the theory of fixed points in a fuzzy context.

Khan et al. [21] improved the Banach fixed point theorem in metric spaces by introducing a control function, called an altering distance function. As altering distance is used a monotone, increasing, and continuous function $\varphi:[0, \infty) \rightarrow[0, \infty)$ such that $\varphi(t)=0$ if and only if $t=0$. In [21] the Banach contraction principle in a complete metric space $(X, d)$ is generalized by condition

$$
\varphi(d(f x, f y)) \leq a \varphi(d(x, y))
$$

for all $x, y \in X$ and some $0<a<1$, where $f: X \rightarrow X$. This result was a motivation for further studies in metric spaces, as well as in the probabilistic metric space [22, 23]. Recently, Shen

(C) 2015 Wairojjana et al.; licensee Springer. This article is distributed under the terms of the Creative Commons Attribution 4.0 International License (http://creativecommons.org/licenses/by/4.0/), which permits unrestricted use, distribution, and reproduction in any medium, provided you give appropriate credit to the original author(s) and the source, provide a link to the Creative Commons license, and indicate if changes were made. 
et al. [24] introduced the notion of altering distance in fuzzy metric space $(X, M, T)$ and by using the contraction condition

$$
\varphi(M(f x, f y, t)) \leq k(t) \cdot \varphi(M(x, y, t)), \quad x, y \in X, x \neq y, t>0,
$$

obtained fixed point results for $f: X \rightarrow X$. Using the same altering distance in this paper several fixed point results are proved in complete and compact fuzzy metric spaces introducing stronger contraction conditions than (1). Likewise, the contraction condition given in [25] is improved by using the altering distance, as well as by extending the class of $t$-norms. Moreover, appropriate examples are presented.

First, the basic definitions and facts are reviewed.

Definition 1.1 A mapping $T:[0,1] \times[0,1] \rightarrow[0,1]$ is called a triangular norm $(t$-norm) if the following conditions are satisfied:

(T1) $T(a, 1)=a, a \in[0,1]$

(T2) $T(a, b)=T(b, a), a, b \in[0,1]$,

(T3) $a \geq b, c \geq d \Rightarrow T(a, c) \geq T(b, d), a, b, c, d \in[0,1]$,

(T4) $T(a, T(b, c))=T(T(a, b), c), a, b, c \in[0,1]$.

Basic examples are $T_{P}(x, y)=x \cdot y, T_{M}(x, y)=\min \{x, y\}, T_{L}(x, y)=\max \{x+y-1,0\}$, and

$$
T_{D}(x, y)= \begin{cases}\min (x, y), & \max (x, y)=1 \\ 0, & \text { otherwise }\end{cases}
$$

Definition 1.2 [26] A $t$-norm $T$ is said to be positive if $T(a, b)>0$ whenever $a, b \in(0,1]$.

Definition 1.3 [2,3] The 3-tuple $(X, M, T)$ is said to be a fuzzy metric space if $X$ is an arbitrary set, $T$ is a continuous $t$-norm, and $M$ is a fuzzy set on $X^{2} \times(0, \infty)$ such that the following conditions are satisfied:

(FM1) $M(x, y, t)>0, x, y \in X, t>0$,

(FM2) $M(x, y, t)=1, t>0 \Leftrightarrow x=y$,

(FM3) $M(x, y, t)=M(y, x, t), x, y \in X, t>0$,

(FM4) $T(M(x, y, t), M(y, z, s)) \leq M(x, z, t+s), x, y, z \in X, t, s>0$,

(FM5) $M(x, y, \cdot):(0, \infty) \rightarrow[0,1]$ is continuous for every $x, y \in X$.

If (FM4) is replaced by condition

(FM4') $T(M(x, y, t), M(y, z, t)) \leq M(x, z, t), x, y, z \in X, t>0$,

then $(X, M, T)$ is called a strong fuzzy metric space [27].

Moreover, if $(X, M, T)$ is a fuzzy metric space, then $M$ is a continuous function on $X \times$ $X \times(0, \infty)[28]$ and $M(x, y, \cdot)$ is non-decreasing for all $x, y \in X[29]$.

If $(X, M, T)$ is a fuzzy metric space, then $M$ generates the Hausdorff topology on $X$ (see $[2,3])$ with a base of open sets $\{U(x, r, t): x \in X, r \in(0,1), t>0\}$, where $U(x, r, t)=\{y: y \in$ $X, M(x, y, t)>1-r\}$.

Definition 1.4 [2,3] Let $(X, M, T)$ be a fuzzy metric space. 
(a) A sequence $\left\{x_{n}\right\}_{n \in \mathbb{N}}$ is a Cauchy sequence in $(X, M, T)$ if for each $\varepsilon \in(0,1)$ and each $t>0$ there exists $n_{0}=n_{0}(\varepsilon, t) \in \mathbb{N}$ such that $M\left(x_{n}, x_{m}, t\right)>1-\varepsilon$, for all $n, m \geq n_{0}$.

(b) A sequence $\left\{x_{n}\right\}_{n \in \mathbb{N}}$ converges to $x$ in $(X, M, T)$ if for each $\varepsilon \in(0,1)$ and each $t>0$ there exists $n_{0}=n_{0}(\varepsilon, t) \in \mathbb{N}$ such that $M\left(x_{n}, x, t\right)>1-\varepsilon$ for all $n \geq n_{0}$. Then we say that $\left\{x_{n}\right\}_{n \in \mathbb{N}}$ is convergent.

(c) A fuzzy metric space $(X, M, T)$ is complete if every Cauchy sequence in $(X, M, T)$ is convergent.

(d) A fuzzy metric space is compact if every sequence in $X$ has a convergent subsequence.

It is well known [2] that in a fuzzy metric space every compact set is closed and bounded.

Definition 1.5 [8] Let $T$ be a $t$-norm and $T_{n}:[0,1] \rightarrow[0,1], n \in \mathbb{N}$, be defined in the following way:

$$
T_{1}(x)=T(x, x), \quad T_{n+1}(x)=T\left(T_{n}(x), x\right), \quad n \in \mathbb{N}, x \in[0,1] .
$$

We say that the $t$-norm $T$ is of $H$-type if the family $\left\{T_{n}(x)\right\}_{n \in \mathbb{N}}$ is equi-continuous at $x=1$.

Each $t$-norm $T$ can be extended (see [26]) (by associativity) in a unique way to an $n$-ary operation taking for $\left(x_{1}, \ldots, x_{n}\right) \in[0,1]^{n}$ the values

$$
\mathbf{T}_{i=1}^{0} x_{i}=1, \quad \mathbf{T}_{i=1}^{n} x_{i}=T\left(\mathbf{T}_{i=1}^{n-1} x_{i}, x_{n}\right)
$$

A $t$-norm $T$ can be extended to a countable infinite operation taking for any sequence $\left(x_{n}\right)_{n \in \mathbb{N}}$ from $[0,1]$ the value

$$
\mathbf{T}_{i=1}^{\infty} x_{i}=\lim _{n \rightarrow \infty} \mathbf{T}_{i=1}^{n} x_{i}
$$

The sequence $\left(\mathbf{T}_{i=1}^{n} x_{i}\right)_{n \in \mathbb{N}}$ is non-increasing and bounded from below, hence the limit $\mathbf{T}_{i=1}^{\infty} x_{i}$ exists.

In the fixed point theory (see $[10,30])$ it is of interest to investigate the classes of $t$-norms $T$ and sequences $\left(x_{n}\right)$ from the interval $[0,1]$ such that $\lim _{n \rightarrow \infty} x_{n}=1$ and

$$
\lim _{n \rightarrow \infty} \mathbf{T}_{i=n}^{\infty} x_{i}=\lim _{n \rightarrow \infty} \mathbf{T}_{i=1}^{\infty} x_{n+i}=1
$$

It is obvious that

$$
\lim _{n \rightarrow \infty} \mathbf{T}_{i=n}^{\infty} x_{i}=1 \quad \Leftrightarrow \quad \sum_{i=1}^{\infty}\left(1-x_{i}\right)<\infty
$$

for $T=T_{L}$ and $T=T_{P}$.

For $T \geq T_{L}$ we have the following implication:

$$
\lim _{n \rightarrow \infty} \mathbf{T}_{i=n}^{\infty} x_{i}=1 \Rightarrow \sum_{i=1}^{\infty}\left(1-x_{i}\right)<\infty .
$$

Example 1.6 Some of the important classes of $t$-norms are the following. 
(i) The Dombi family of $t$-norms $\left(T_{\lambda}^{\mathrm{D}}\right)_{\lambda \in[0, \infty]}$ is defined by

$$
T_{\lambda}^{\mathrm{D}}(x, y)= \begin{cases}T_{D}(x, y), & \lambda=0, \\ T_{M}(x, y), & \lambda=\infty, \\ \frac{1}{1+\left(\left(\frac{1-x}{x}\right)^{\lambda}+\left(\frac{1-y}{y}\right)^{\lambda}\right)^{1 / \lambda},} & \lambda \in(0, \infty) .\end{cases}
$$

(ii) The Aczél-Alsina family of $t$-norms $\left(T_{\lambda}^{\mathrm{AA}}\right)_{\lambda \in[0, \infty]}$ is defined by

$$
T_{\lambda}^{\mathrm{AA}}(x, y)= \begin{cases}T_{D}(x, y), & \lambda=0, \\ T_{M}(x, y), & \lambda=\infty, \\ e^{-\left((-\log x)^{\lambda}+(-\log y)^{\lambda}\right)^{1 / \lambda},} & \lambda \in(0, \infty) .\end{cases}
$$

(iii) The family $\left(T_{\lambda}^{\mathrm{SW}}\right)_{\lambda \in[-1, \infty]}$ of Sugeno-Weber $t$-norms is given by

$$
T_{\lambda}^{\mathrm{SW}}(x, y)= \begin{cases}T_{D}(x, y), & \lambda=-1, \\ T_{P}(x, y), & \lambda=\infty, \\ \max \left(0, \frac{x+y-1+\lambda x y}{1+\lambda}\right), & \lambda \in(-1, \infty) .\end{cases}
$$

(iv) The Schweizer-Sklar family of $t$-norms $\left(T_{\lambda}^{\mathrm{SS}}\right)_{\lambda \in[-\infty, \infty]}$ is defined by

$$
T_{\lambda}^{S S}(x, y)= \begin{cases}T_{M}(x, y), & \lambda=-\infty, \\ T_{P}(x, y), & \lambda=0, \\ \left(\max \left(x^{\lambda}+y^{\lambda}-1,0\right)\right)^{1 / \lambda}, & \lambda \in(-\infty, 0) \cup(0, \infty), \\ T_{D}(x, y), & \lambda=\infty .\end{cases}
$$

The condition $T \geq T_{L}$ is fulfilled by the families $\left(T_{\lambda}^{\mathrm{SS}}\right)_{\lambda \in(-\infty, 1)},\left(T_{\lambda}^{\mathrm{SW}}\right)_{\lambda \in[0, \infty]}$.

On the other side, there exists a member of the family $\left(T_{\lambda}^{\mathrm{D}}\right)_{\lambda \in(0, \infty)}$ which is incomparable with $T_{L}$, and there exists a member of the family $\left(T_{\lambda}^{\mathrm{AA}}\right)_{\lambda \in(0, \infty)}$ which is incomparable with $T_{L}$.

In [10] the following results are obtained.

(a) If $\left(T_{\lambda}^{\mathrm{D}}\right)_{\lambda \in(0, \infty)}$ is the Dombi family of $t$-norms and $\left(x_{n}\right)_{n \in \mathbb{N}}$ a sequence of elements from $(0,1]$ such that $\lim _{n \rightarrow \infty} x_{n}=1$, then we have the following equivalence:

$$
\sum_{n=1}^{\infty}\left(1-x_{n}\right)^{\lambda}<\infty \quad \Leftrightarrow \quad \lim _{n \rightarrow \infty}\left(T_{\lambda}^{\mathrm{D}}\right)_{i=n}^{\infty} x_{i}=1
$$

(b) If $\left(T_{\lambda}^{\mathrm{SW}}\right)_{\lambda \in(-1, \infty]}$ is the Sugeno-Weber family of $t$-norms and $\left(x_{n}\right)_{n \in \mathbb{N}}$ a sequence of elements from $(0,1]$ such that $\lim _{n \rightarrow \infty} x_{n}=1$, then we have the following equivalence:

$$
\sum_{n=1}^{\infty}\left(1-x_{n}\right)<\infty \Leftrightarrow \lim _{n \rightarrow \infty}\left(T_{\lambda}^{\mathrm{SW}}\right)_{i=n}^{\infty} x_{i}=1
$$

(c) The equivalence (2) holds also for the family $\left(T_{\lambda}^{\mathrm{AA}}\right)_{\lambda \in(0, \infty)}$, i.e.

$$
\sum_{n=1}^{\infty}\left(1-x_{n}\right)^{\lambda}<\infty \Leftrightarrow \lim _{n \rightarrow \infty}\left(T_{\lambda}^{\mathrm{AA}}\right)_{i=n}^{\infty} x_{i}=1
$$

In [10] the following proposition is obtained. 
Proposition 1.7 Let $\left(x_{n}\right)_{n \in \mathbb{N}}$ be a sequence of numbers from $[0,1]$ such that $\lim _{n \rightarrow \infty} x_{n}=1$ and the t-norm $T$ is of H-type. Then $\lim _{n \rightarrow \infty} \mathbf{T}_{i=n}^{\infty} x_{i}=\lim _{n \rightarrow \infty} \mathbf{T}_{i=1}^{\infty} x_{n+i}=1$.

Theorem 1.8 [31] Let $(X, M, T)$ be a fuzzy metric space, such that $\lim _{t \rightarrow \infty} M(x, y, t)=1$. Then iffor some $\sigma_{0} \in(0,1)$ and some $x_{0}, y_{0} \in X$ the following hold:

$$
\lim _{n \rightarrow \infty} \mathbf{T}_{i=n}^{\infty} M\left(x_{0}, y_{0}, \frac{1}{\sigma_{0}^{i}}\right)=1
$$

then

$$
\lim _{n \rightarrow \infty} \mathbf{T}_{i=n}^{\infty} M\left(x_{0}, y_{0}, \frac{1}{\sigma^{i}}\right)=1
$$

for every $\sigma \in(0,1)$.

\section{Main results}

A function $\varphi:[0,1] \rightarrow[0,1]$ is called an altering distance function $[23,24]$ if it satisfies the following properties:

(AD1) $\varphi$ is strictly decreasing and continuous;

(AD2) $\varphi(\lambda)=0$ if and only if $\lambda=1$.

It is obvious that $\lim _{\lambda \rightarrow 1^{-}} \varphi(\lambda)=\varphi(1)=0$.

Theorem 2.1 Let $(X, M, T)$ be a complete fuzzy metric space, $T$ be a triangular norm and $f: X \rightarrow X$. If there exist $k_{1}, k_{2}:(0, \infty) \rightarrow(0,1)$, and an altering distance function $\varphi$ such that the following condition:

$$
\begin{aligned}
\varphi(M(f x, f y, t)) \leq & k_{1}(t) \cdot \min \{\varphi(M(x, y, t)), \varphi(M(x, f x, t)), \varphi(M(x, f y, 2 t)), \varphi(M(y, f y, t))\} \\
& +k_{2}(t) \cdot \varphi(M(f x, y, 2 t)), \quad x, y \in X, x \neq y, t>0,
\end{aligned}
$$

is satisfied, then $f$ has a unique fixed point.

Proof We observe a sequence $\left\{x_{n}\right\}$, where $x_{0} \in X$ and $x_{n+1}=f x_{n}, n \in \mathbb{N} \cup\{0\}$. Note that, if there exists $n_{0} \in \mathbb{N} \cup\{0\}$ such that $x_{n_{0}}=x_{n_{0}+1}=f x_{n_{0}}$, then $x_{n_{0}}$ is a fixed point of $f$. Further, we assume that $x_{n} \neq x_{n+1}, n \in \mathbb{N}_{0}$. Then

$$
0<M\left(x_{n}, x_{n+1}, t\right)<1, \quad t>0, n \in \mathbb{N}_{0} .
$$

If the pair $x=x_{n-1}, y=x_{n}$ satisfy condition (6) then

$$
\begin{aligned}
\varphi\left(M\left(x_{n}, x_{n+1}, t\right)\right) \leq & k_{1}(t) \cdot \min \left\{\varphi\left(M\left(x_{n-1}, x_{n}, t\right)\right), \varphi\left(M\left(x_{n-1}, x_{n}, t\right)\right), \varphi\left(M\left(x_{n-1}, x_{n+1}, 2 t\right)\right),\right. \\
& \left.\varphi\left(M\left(x_{n}, x_{n+1}, t\right)\right)\right\}+k_{2}(t) \cdot \varphi\left(M\left(x_{n}, x_{n}, 2 t\right)\right) \\
= & k_{1}(t) \cdot \min \left\{\varphi\left(M\left(x_{n-1}, x_{n}, t\right)\right), \varphi\left(M\left(x_{n-1}, x_{n+1}, 2 t\right)\right)\right. \\
& \left.\varphi\left(M\left(x_{n}, x_{n+1}, t\right)\right)\right\}, \quad n \in \mathbb{N}, t>0 .
\end{aligned}
$$

By (FM4) and (AD1) we have

$$
\varphi\left(M\left(x_{n-1}, x_{n+1}, 2 t\right)\right) \leq \varphi\left(T\left(M\left(x_{n-1}, x_{n}, t\right), M\left(x_{n}, x_{n+1}, t\right)\right)\right), \quad n \in \mathbb{N}, t>0,
$$


and further

$$
\begin{aligned}
\varphi\left(M\left(x_{n}, x_{n+1}, t\right)\right) \leq & k_{1}(t) \cdot \min \left\{\varphi\left(M\left(x_{n-1}, x_{n}, t\right)\right), \varphi\left(T \left(M\left(x_{n-1}, x_{n}, t\right),\right.\right.\right. \\
& \left.\left.\left.M\left(x_{n}, x_{n+1}, t\right)\right)\right), \varphi\left(M\left(x_{n}, x_{n+1}, t\right)\right)\right\}, \quad n \in \mathbb{N}, t>0 .
\end{aligned}
$$

Note that, by (T1), (T2), and (T3), it follows that

$$
a=T(a, 1) \geq T(a, b) \quad \text { and } \quad b=T(b, 1) \geq T(a, b)
$$

and

$$
\min \{\varphi(a), \varphi(b), \varphi(T(a, b))\}=\min \{\varphi(a), \varphi(b)\}, \quad a, b \in[0,1] .
$$

Then by (9) we have

$$
\varphi\left(M\left(x_{n}, x_{n+1}, t\right)\right) \leq k_{1}(t) \cdot \min \left\{\varphi\left(M\left(x_{n-1}, x_{n}, t\right)\right), \varphi\left(M\left(x_{n}, x_{n+1}, t\right)\right)\right\}, \quad n \in \mathbb{N}, t>0
$$

If we suppose that

$$
\min \left\{\varphi\left(M\left(x_{n-1}, x_{n}, t\right)\right), \varphi\left(M\left(x_{n}, x_{n+1}, t\right)\right)\right\}=\varphi\left(M\left(x_{n}, x_{n+1}, t\right)\right), \quad n \in \mathbb{N}, t>0,
$$

then

$$
\varphi\left(M\left(x_{n}, x_{n+1}, t\right)\right) \leq k_{1}(t) \cdot \varphi\left(M\left(x_{n}, x_{n+1}, t\right)\right)<\varphi\left(M\left(x_{n}, x_{n+1}, t\right)\right), \quad n \in \mathbb{N}, t>0 .
$$

So, by contradiction it follows that

$$
\min \left\{\varphi\left(M\left(x_{n-1}, x_{n}, t\right)\right), \varphi\left(M\left(x_{n}, x_{n+1}, t\right)\right)\right\}=\varphi\left(M\left(x_{n-1}, x_{n}, t\right)\right), \quad n \in \mathbb{N}, t>0,
$$

and by (10) we get

$$
\varphi\left(M\left(x_{n}, x_{n+1}, t\right)\right) \leq k_{1}(t) \cdot \varphi\left(M\left(x_{n-1}, x_{n}, t\right)\right)<\varphi\left(M\left(x_{n-1}, x_{n}, t\right)\right), \quad n \in \mathbb{N}, t>0 .
$$

By (AD1) and (11) it follows that the sequence $\left\{M\left(x_{n}, x_{n+1}, t\right)\right\}$ is strictly increasing with respect to $n$, for every $t>0$. This fact, together with (7), implies that

$$
\lim _{n \rightarrow \infty} M\left(x_{n}, x_{n+1}, t\right)=a(t), \quad a:(0, \infty) \rightarrow[0,1] .
$$

But if we suppose that $a(t) \neq 1$, for some $t>0$, and let $n \rightarrow \infty$ in (11) we get a contradiction:

$$
\varphi(a(t)) \leq k_{1}(t) \cdot \varphi(a(t))<\varphi(a(t))
$$

So, $a \equiv 1$ in (12). Now we will show that the sequence $\left\{x_{n}\right\}$ is a Cauchy sequence. Suppose the contrary, i.e. that there exist $0<\varepsilon<1, t>0$, and two sequences of integers $\{p(n)\}$, $\{q(n)\}, p(n)>q(n)>n, n \in \mathbb{N} \cup\{0\}$, such that

$$
M\left(x_{p(n)}, x_{q(n)}, t\right) \leq 1-\varepsilon \quad \text { and } \quad M\left(x_{p(n)-1}, x_{q(n)}, t\right)>1-\varepsilon .
$$


By (12) for every $\varepsilon_{1}, 0<\varepsilon_{1}<\varepsilon$, it is possible to find a positive integer $n_{1}$, such that for all $n>n_{1}$,

$$
M\left(x_{p(n)}, x_{p(n)-1}, t\right) \geq 1-\varepsilon_{1} \quad \text { and } \quad M\left(x_{q(n)}, x_{q(n)-1}, t\right) \geq 1-\varepsilon_{1} .
$$

Then we have

$$
M\left(x_{p(n)-1}, x_{q(n)-1}, t\right) \geq T\left(M\left(x_{p(n)-1}, x_{q(n)}, \frac{t}{2}\right), M\left(x_{q(n)}, x_{q(n)-1}, \frac{t}{2}\right)\right), \quad n \in \mathbb{N} .
$$

Now using (14), (15), (16), and (T3) we have

$$
M\left(x_{p(n)-1}, x_{q(n)-1}, t\right) \geq T\left(1-\varepsilon, 1-\varepsilon_{1}\right), \quad n>n_{1} .
$$

Since $\varepsilon_{1}$ is arbitrary and $T$ is continuous we have

$$
M\left(x_{p(n)-1}, x_{q(n)-1}, t\right) \geq T(1-\varepsilon, 1)=1-\varepsilon, \quad n>n_{1} .
$$

Similarly, by (15) and (18)

$$
M\left(x_{p(n)}, x_{q(n)-1}, t\right) \geq 1-\varepsilon \quad \text { and } \quad M\left(x_{p(n)}, x_{q(n)}, t\right) \geq 1-\varepsilon, \quad n>n_{1} .
$$

By (14) and (19) it follows that

$$
\lim _{n \rightarrow \infty} M\left(x_{p(n)}, x_{q(n)}, t\right)=1-\varepsilon
$$

If the pair $x=x_{p(n)-1}, y=x_{q(n)-1}$ satisfy condition (6) then

$$
\begin{aligned}
\varphi\left(M\left(x_{p(n)}, x_{q(n)}, t\right)\right) \leq & k_{1}(t) \cdot \min \left\{\varphi\left(M\left(x_{p(n)-1}, x_{q(n)-1}, t\right)\right), \varphi\left(M\left(x_{p(n)-1}, x_{p(n)}, t\right)\right)\right. \\
& \left.\varphi\left(M\left(x_{p(n)-1}, x_{q(n)}, 2 t\right)\right), \varphi\left(M\left(x_{q(n)-1}, x_{q(n)}, t\right)\right)\right\} \\
& +k_{2}(t) \cdot \varphi\left(M\left(x_{p(n)}, x_{q(n)-1}, 2 t\right)\right) .
\end{aligned}
$$

Letting $n \rightarrow \infty$, by (12), (14), (19), and (20), we have

$$
\varphi(1-\varepsilon) \leq k_{1}(t) \cdot \min \{\varphi(1-\varepsilon), \varphi(1), \varphi(1-\varepsilon), \varphi(1)\}+k_{2}(t) \cdot \varphi(1-\varepsilon)<\varphi(1-\varepsilon) .
$$

This is a contradiction. So $\left\{x_{n}\right\}$ is a Cauchy sequence.

Since $(X, M, T)$ is a complete fuzzy metric space there exists $x \in X$ such that $\lim _{n \rightarrow \infty} x_{n}=x$. Let us prove, by contradiction, that $x$ is fixed point for $f$. Suppose that $x \neq f x$.

If the pair $x=x, y=x_{n-1}$ satisfy condition (6) then

$$
\begin{aligned}
\varphi\left(M\left(f x, x_{n}, t\right)\right) \leq & k_{1}(t) \cdot \min \left\{\varphi\left(M\left(x, x_{n-1}, t\right)\right), \varphi(M(x, f x, t)), \varphi\left(M\left(x, x_{n}, 2 t\right)\right),\right. \\
& \left.\varphi\left(M\left(x_{n-1}, x_{n}, t\right)\right)\right\}+k_{2}(t) \cdot \varphi\left(M\left(f x, x_{n-1}, 2 t\right)\right), \quad n \in \mathbb{N}, t>0 .
\end{aligned}
$$


Letting $n \rightarrow \infty$ in (23) we have

$$
\begin{aligned}
\varphi(M(f x, x, t)) \leq & k_{1}(t) \cdot \min \{\varphi(M(x, x, t)), \varphi(M(x, f x, t)), \varphi(M(x, x, 2 t))\} \\
& +k_{2}(t) \cdot \varphi(M(f x, x, 2 t))<\varphi(M(f x, x, 2 t))<\varphi(M(f x, x, t)), \quad t>0 .
\end{aligned}
$$

So, by contradiction we conclude that $x=f x$.

Assume now that there exists another fixed point $v, v \neq x$. Then applying (6) we have

$$
\begin{aligned}
\varphi(M(x, v, t)) \leq & k_{1}(t) \cdot \min \{\varphi(M(x, v, t)), \varphi(M(x, x, t)), \varphi(M(x, v, 2 t)), \varphi(M(v, v, t))\} \\
& +k_{2}(t) \cdot \varphi(M(x, v, 2 t))<\varphi(M(x, v, 2 t))<\varphi(M(x, v, t)), \quad t>0 .
\end{aligned}
$$

So, we get a contradiction, and $x$ is a unique fixed point of the function $f$.

Example 2.2 Let $X=\{A, B, C, D\}$ be subset of $\mathbb{R}^{2}$, where $A(0,0), B(1,0), C(1,4)$. Let $D$ be an arbitrary point on circle of radius 1 with center in $C$. Let $f: X \rightarrow X$ be defined by

$$
f(A)=f(B)=f(D)=A, \quad f(C)=B .
$$

Let $\varphi(\tau)=1-\tau, \tau \in[0,1]$, and $M(x, y, t)=\frac{t}{t+d(x, y)}, t>0$, where by $d(x, y)$ is denoted the Euclidean distance in $\mathbb{R}^{2}$. Note that by $(X, M, T)$ is defined a complete fuzzy metric space with respect to the triangular norm $T(a, b)=\min \{a, b\}$ and $\varphi$ satisfies conditions (AD1) and (AD2). The functions $k_{1}, k_{2}:(0, \infty) \rightarrow(0,1)$ are defined by

$$
k_{1}(t)=k_{2}(t)= \begin{cases}\frac{e^{2}}{t+e^{2}}, & t \in(0,1], \\ \frac{t}{t+\frac{1}{e^{2}}}, & t \in(1, \infty) .\end{cases}
$$

Now, every pair of points $x, y \in X, x \neq y$, satisfy condition (6) and by Theorem 2.1 it follows that $f$ has a unique fixed point. On the other side, for $x=C$ and $y=D$ condition (1) given in [24] as a classical generalization of the Banach principle of contraction is not satisfied. Nevertheless, the condition (6) is not a full generalization of condition (1).

Theorem 2.3 Let $(X, M, T)$ be a complete fuzzy metric space and $f: X \rightarrow X$. If there exist $k_{1}, k_{2}:(0, \infty) \rightarrow[0,1), k_{3}:(0, \infty) \rightarrow(0,1), \sum_{i=1}^{3} k_{i}(t)<1$, and an altering distance function $\varphi$ such that the following condition is satisfied:

$$
\varphi(M(f x, f y, t)) \leq k_{1}(t) \cdot \varphi(M(x, f x, t))+k_{2}(t) \cdot \varphi(M(y, f y, t))+k_{3}(t) \cdot \varphi(M(x, y, t))
$$

for all $x, y \in X, x \neq y$, and $t>0$, then $f$ has a unique fixed point.

Proof Let $x_{0} \in X$ and $x_{n+1}=f x_{n}$. Suppose that $x_{n} \neq x_{n+1}, n \in \mathbb{N}_{0}$, i.e.

$$
0<M\left(x_{n}, x_{n+1}, t\right)<1, \quad n \in \mathbb{N}_{0}, t>0 .
$$

By (25), with $x=x_{n-1}, y=x_{n}$, we have

$$
\begin{aligned}
\varphi\left(M\left(x_{n}, x_{n+1}, t\right)\right) \leq & k_{1}(t) \cdot \varphi\left(M\left(x_{n-1}, x_{n}, t\right)\right)+k_{2}(t) \cdot \varphi\left(M\left(x_{n}, x_{n+1}, t\right)\right) \\
& +k_{3}(t) \cdot \varphi\left(M\left(x_{n-1}, x_{n}, t\right)\right), \quad n \in \mathbb{N}_{0}, t>0,
\end{aligned}
$$


i.e.

$$
\begin{aligned}
\varphi\left(M\left(x_{n}, x_{n+1}, t\right)\right) & \leq \frac{k_{1}(t)+k_{3}(t)}{1-k_{2}(t)} \cdot \varphi\left(M\left(x_{n-1}, x_{n}, t\right)\right) \\
& <\varphi\left(M\left(x_{n-1}, x_{n}, t\right)\right), \quad n \in \mathbb{N}_{0}, t>0 .
\end{aligned}
$$

Since $\varphi$ is strictly decreasing, the sequence $\left\{M\left(x_{n}, x_{n+1}, t\right)\right\}$ is strictly increasing sequence, with respect to $n$, for every $t>0$. Hence, by (26) and a similar method to Theorem 2.1 it could be shown that

$$
\lim _{n \rightarrow \infty} M\left(x_{n}, x_{n+1}, t\right)=1, \quad t>0,
$$

and $\left\{x_{n}\right\}$ is a Cauchy sequence. So, there exists $x \in X$ such that $\lim _{n \rightarrow \infty} x_{n}=x$. Now, by (25) with $x=x_{n-1}, y=x$ we have

$$
\begin{aligned}
\varphi\left(M\left(x_{n}, f x, t\right)\right) \leq & k_{1}(t) \cdot \varphi\left(M\left(x_{n-1}, x_{n}, t\right)\right)+k_{2}(t) \cdot \varphi(M(x, f x, t)) \\
& +k_{3}(t) \cdot \varphi\left(M\left(x_{n-1}, x, t\right)\right),
\end{aligned}
$$

$n \in \mathbb{N}, t>0$. Letting $n \rightarrow \infty$ in (30) we have

$$
\begin{aligned}
\varphi(M(x, f x, t)) & \leq k_{1}(t) \cdot \varphi(1)+k_{2}(t) \cdot \varphi(M(x, f x, t))+k_{3}(t) \cdot \varphi(1) \\
& =k_{2}(t) \cdot \varphi(M(x, f x, t)), \quad t>0,
\end{aligned}
$$

i.e.

$$
\left(1-k_{2}(t)\right) \varphi(M(x, f x, t)) \leq 0, \quad t>0 .
$$

It follows that $\varphi(M(x, f x, t))=0$ and $x=f x$.

Assume now that there exists a fixed point $v, v \neq x$. Then by (25) we have

$$
\begin{aligned}
\varphi(M(f x, f v, t)) \leq & k_{1}(t) \cdot \varphi(M(x, f x, t))+k_{2}(t) \cdot \varphi(M(v, f v, t)) \\
& +k_{3}(t) \cdot \varphi(M(x, v, t))<\varphi(M(x, v, t)), \quad t>0,
\end{aligned}
$$

which is a contradiction. So, $x$ is a unique fixed point of $f$.

Remark 2.4 If in (25) we take $k_{1}(t)=k_{2}(t)=0, t>0$, we get condition (1), and Theorem 2.3. is a generalization of the result given in [24].

In the following theorems conditions (34) and (47) proposed in [32] are used to obtain fixed point results in complete and compact strong fuzzy metric spaces.

Theorem 2.5 Let $(X, M, T)$ be a complete strongfuzzy metric space with positive t-norm $T$ and let $f: X \rightarrow X$. If there exists an altering distance function $\varphi$ and $a_{i}=a_{i}(t), i=1,2, \ldots, 5$, $a_{i}>0, a_{1}+a_{2}+2 a_{3}+2 a_{4}+a_{5}<1$, such that

$$
\varphi(T(r, s)) \leq \varphi(r)+\varphi(s), \quad r, s \in\{M(x, f x, t): x \in X, t>0\}
$$


and

$$
\begin{aligned}
\varphi(M(f x, f y, t)) \leq & a_{1} \varphi(M(f x, x, t))+a_{2} \varphi(M(f y, y, t))+a_{3} \varphi(M(f x, y, t)) \\
& +a_{4} \varphi(M(x, f y, t))+a_{5} \varphi(M(x, y, t)), \quad x, y \in X, t>0,
\end{aligned}
$$

then $f$ has a unique fixed point.

Proof Let $x_{0} \in X$ be arbitrary. Define a sequence $\left(x_{n}\right)_{n \in \mathbb{N}}$ such that $x_{n}=f x_{n-1}=f^{n} x_{0}$. By (35) with $x=x_{n-1}$ and $y=x_{n}$ we have

$$
\begin{aligned}
\varphi\left(M\left(x_{n}, x_{n+1}, t\right)\right) \leq & a_{1} \varphi\left(M\left(x_{n}, x_{n-1}, t\right)\right)+a_{2} \varphi\left(M\left(x_{n+1}, x_{n}, t\right)\right)+a_{3} \varphi\left(M\left(x_{n}, x_{n}, t\right)\right) \\
& +a_{4} \varphi\left(M\left(x_{n-1}, x_{n+1}, t\right)\right)+a_{5} \varphi\left(M\left(x_{n-1}, x_{n}, t\right)\right), \quad n \in \mathbb{N}, t>0
\end{aligned}
$$

Since $(X, M, T)$ is a strong fuzzy metric space we have

$$
M\left(x_{n-1}, x_{n+1}, t\right) \geq T\left(M\left(x_{n-1}, x_{n}, t\right), M\left(x_{n}, x_{n+1}, t\right)\right), \quad n \in \mathbb{N}, t>0,
$$

using (34) we obtained

$$
\begin{aligned}
\varphi\left(M\left(x_{n-1}, x_{n+1}, t\right)\right) & \leq \varphi\left(T\left(M\left(x_{n-1}, x_{n}, t\right), M\left(x_{n}, x_{n+1}, t\right)\right)\right) \\
& \leq \varphi\left(M\left(x_{n-1}, x_{n}, t\right)\right)+\varphi\left(M\left(x_{n}, x_{n+1}, t\right)\right), \quad n \in \mathbb{N}, t>0 .
\end{aligned}
$$

By (36) it follows that

$$
\varphi\left(M\left(x_{n}, x_{n+1}, t\right)\right) \leq \frac{a_{1}+a_{4}+a_{5}}{1-a_{2}-a_{4}} \varphi\left(M\left(x_{n-1}, x_{n}, t\right)\right)<\varphi\left(M\left(x_{n-1}, x_{n}, t\right)\right), \quad n \in \mathbb{N}, t>0
$$

i.e.

$$
M\left(x_{n}, x_{n+1}, t\right)>M\left(x_{n-1}, x_{n}, t\right), \quad n \in \mathbb{N}, t>0 .
$$

So, the sequence $\left\{M\left(x_{n}, x_{n+1}, t\right)\right\}$ is increasing and bounded and there exists

$$
\lim _{n \rightarrow \infty} M\left(x_{n}, x_{n+1}, t\right)=p(t), \quad p:(0, \infty) \rightarrow[0,1]
$$

Suppose that $p(t) \neq 1$, for some $t>0$. Then, if we take $n \rightarrow \infty$ in (36)

$$
\varphi(p(t)) \leq\left(a_{1}+a_{2}+2 a_{4}+a_{5}\right) \varphi(p(t))<\varphi(p(t))
$$

and we get a contradiction, i.e.

$$
\lim _{n \rightarrow \infty} M\left(x_{n}, x_{n+1}, t\right)=1, \quad t>0 .
$$

It remains to prove that $\left\{x_{n}\right\}_{n \in \mathbb{N}}$ is a Cauchy sequence. Suppose the contrary, i.e. that there exist $\varepsilon>0, t>0$, such that for every $s \in \mathbb{N}$ there exist $m(s)>n(s) \geq s$, and

$$
M\left(x_{m(s)}, x_{n(s)}, t\right)<1-\varepsilon .
$$


Let $m(s)$ be the least integer exceeding $n(s)$ satisfying the above property, i.e.

$$
M\left(x_{m(s)-1}, x_{n(s)}, t\right) \geq 1-\varepsilon, \quad s \in \mathbb{N}, t>0 .
$$

Then by (35) with $x=x_{m(s)-1}$ and $y=x_{n(s)-1}$, for each $s \in \mathbb{N}$ and $t>0$ we have

$$
\begin{aligned}
\varphi\left(M\left(x_{m(s)}, x_{n(s)}, t\right)\right) \leq & a_{1} \varphi\left(M\left(x_{m(s)}, x_{m(s)-1}, t\right)\right)+a_{2} \varphi\left(M\left(x_{n(s)}, x_{n(s)-1}, t\right)\right) \\
& +a_{3} \varphi\left(M\left(x_{m(s)}, x_{n(s)-1}, t\right)\right)+a_{4} \varphi\left(M\left(x_{m(s)-1}, x_{n(s)}, t\right)\right) \\
& +a_{5} \varphi\left(M\left(x_{m(s)-1}, x_{n(s)-1}, t\right)\right) .
\end{aligned}
$$

By (FM4'), (34), and (AD1) it follows that

$$
\varphi\left(M\left(x_{m(s)}, x_{n(s)-1}, t\right)\right) \leq \varphi\left(M\left(x_{m(s)}, x_{n(s)}, t\right)\right)+\varphi\left(M\left(x_{n(s)}, x_{n(s)-1}, t\right)\right)
$$

and

$$
\varphi\left(M\left(x_{m(s)-1}, x_{n(s)-1}, t\right)\right) \leq \varphi\left(M\left(x_{m(s)-1}, x_{m(s)}, t\right)\right)+\varphi\left(M\left(x_{m(s)}, x_{n(s)-1}, t\right)\right) .
$$

Combining the previous inequalities we get

$$
\begin{aligned}
\varphi\left(M\left(x_{m(s)-1}, x_{n(s)-1}, t\right)\right) \leq & \varphi\left(M\left(x_{m(s)-1}, x_{m(s)}, t\right)\right)+\varphi\left(M\left(x_{m(s)}, x_{n(s)}, t\right)\right) \\
& +\varphi\left(M\left(x_{n(s)}, x_{n(s)-1}, t\right)\right) .
\end{aligned}
$$

Also, by (38) and (AD1) we have

$$
\varphi\left(M\left(x_{m(s)-1}, x_{n(s)}, t\right)\right) \leq \varphi(1-\varepsilon) .
$$

Inserting (40), (41), and (42) in (39) we obtain

$$
\begin{aligned}
\varphi\left(M\left(x_{m(s)}, x_{n(s)}, t\right)\right) \leq & a_{1} \varphi\left(M\left(x_{m(s)}, x_{m(s)-1}, t\right)\right)+a_{2} \varphi\left(M\left(x_{n(s)}, x_{n(s)-1}, t\right)\right) \\
& +a_{3} \varphi\left(M\left(x_{m(s)}, x_{n(s)}, t\right)\right)+a_{3} \varphi\left(M\left(x_{n(s)}, x_{n(s)-1}, t\right)\right)+a_{4} \varphi(1-\varepsilon) \\
& +a_{5} \varphi\left(M\left(x_{m(s)}, x_{m(s)-1}, t\right)\right)+a_{5} \varphi\left(M\left(x_{m(s)}, x_{n(s)}, t\right)\right) \\
& +a_{5} \varphi\left(M\left(x_{n(s)}, x_{n(s)-1}, t\right)\right)
\end{aligned}
$$

i.e.

$$
\begin{aligned}
& \left(1-a_{3}-a_{5}\right) \varphi\left(M\left(x_{m(s)}, x_{n(s)}, t\right)\right) \\
& \leq\left(a_{1}+a_{5}\right) \varphi\left(M\left(x_{m(s)}, x_{m(s)-1}, t\right)\right) \\
& \quad+\left(a_{2}+a_{3}+a_{5}\right) \varphi\left(M\left(x_{n(s)}, x_{n(s)-1}, t\right)\right)+a_{4} \varphi(1-\varepsilon) .
\end{aligned}
$$

By (37) it follows that

$$
\varphi\left(M\left(x_{m(s)}, x_{n(s)}, t\right)\right)>\varphi(1-\varepsilon),
$$


and (43) and (44) imply

$$
\begin{aligned}
\left(1-a_{3}-a_{5}\right) \varphi(1-\varepsilon)< & \left(a_{1}+a_{5}\right) \varphi\left(M\left(x_{m(s)}, x_{m(s)-1}, t\right)\right) \\
& +\left(a_{2}+a_{3}+a_{5}\right) \varphi\left(M\left(x_{n(s)}, x_{n(s)-1}, t\right)\right)+a_{4} \varphi(1-\varepsilon) .
\end{aligned}
$$

Letting $s \rightarrow \infty$ in (45) we have

$$
\left(1-a_{3}-a_{5}\right) \varphi(1-\varepsilon) \leq a_{4} \varphi(1-\varepsilon)
$$

i.e.

$$
\left(1-a_{3}-a_{4}-a_{5}\right) \varphi(1-\varepsilon) \leq 0,
$$

which implies that $\varepsilon=0$ and we get a contradiction.

So, $\left\{x_{n}\right\}_{n \in \mathbb{N}}$ is a Cauchy sequence and there exist $z \in X$ such that $\lim _{n \rightarrow \infty} x_{n}=z$. Now, by (35) with $x=x_{n-1}$ and $y=z$, we have

$$
\begin{aligned}
\varphi\left(M\left(x_{n}, f z, t\right)\right) \leq & a_{1} \varphi\left(M\left(x_{n}, x_{n-1}, t\right)\right)+a_{2} \varphi(M(f z, z, t))+a_{3} \varphi\left(M\left(x_{n}, z, t\right)\right) \\
& +a_{4} \varphi\left(M\left(x_{n-1}, f z, t\right)\right)+a_{5} \varphi\left(M\left(x_{n-1}, z, t\right)\right), \quad n \in \mathbb{N}, t>0 .
\end{aligned}
$$

Letting $n \rightarrow \infty$ in (46) we have

$$
\left(1-a_{2}-a_{4}\right) \varphi(M(z, f z, t)) \leq 0, \quad t>0
$$

Therefore, $M(z, f z, t)=1, t>0$, and $z=f z$.

Suppose now that there exists another fixed point $w=f w$. By (35) with $x=z$ and $y=w$ we get

$$
\left(1-a_{3}-a_{4}-a_{5}\right) \varphi(M(z, w, t)) \leq 0, \quad t>0,
$$

i.e. $z=w$.

Example 2.6 Let $X=\{A, B, C, D\}$ be subset of $\mathbb{R}^{2}$, where $A(0,0), B(1,0), C(1,4)$, and $D(0,4)$. Let $f: X \rightarrow X$ be defined by

$$
f(A)=f(B)=f(D)=A, \quad f(C)=B
$$

Let $\varphi(\tau)=1-\tau, \tau \in[0,1]$, and $M(x, y, t)=\frac{t}{t+|x-y|}, t>0$. Note that by $(X, M, T)$ is defined a strong complete fuzzy metric space with respect to the triangular norm $T(a, b)=a \cdot b$ and $\varphi$ satisfies conditions (AD1) and (AD2). The functions $a_{i}(t), i \in\{1,2, \ldots, 5\}$ are defined by

$$
a_{2}(t)=\frac{t+\sqrt{17}}{\sqrt{17}(t+1)}, \quad a_{1}(t)=a_{3}(t)=a_{4}(t)=a_{5}(t)=\frac{1-a_{2}(t)}{7}, \quad t>0 .
$$

Now, every pair of points $x, y \in X, x \neq y$, satisfy condition (35) and by Theorem 2.5 it follows that $f$ has a unique fixed point. Note that, for $x=C$ and $y=D$, condition (1) is not satisfied. 
Theorem 2.7 Let $(X, M, T)$ be a compact strong fuzzy metric space and let $\varphi:[0,1] \rightarrow$ $[0,1]$ satisfying conditions (AD1) and (AD2) such that

$$
\varphi(T(r, s)) \leq \varphi(r)+\varphi(s), \quad r, s \in\{M(x, f x, t): x \in X, t>0\} .
$$

If the continuous mapping $f: X \rightarrow X$ satisfies the following condition:

$$
\begin{aligned}
\varphi(M(f x, f y, t)) \leq & a_{1} \varphi(M(f x, x, t))+a_{2} \varphi(M(f y, y, t))+a_{3} \varphi(M(f x, y, t)) \\
& +a_{4} \varphi(M(x, f y, t))+a_{5} \varphi(M(x, y, t)), \quad x, y \in X, x \neq y, t>0
\end{aligned}
$$

for $a_{i}=a_{i}(x, y, t)>0, i=1,2, \ldots, 5, a_{1}+a_{2}+2 a_{3}+2 a_{4}+a_{5}<1$, then $f$ has a unique fixed point in $X$.

Proof Let $x_{0} \in X$. We define the sequence $x_{n+1}=f x_{n}, n \in \mathbb{N}_{0}$ and suppose that $x_{n+1} \neq x_{n}$, $n \in \mathbb{N}_{0}$. Since $(X, M, T)$ is compact, the sequence $\left\{x_{n}\right\}_{n \in \mathbb{N}_{0}}$ has a subsequence $\left\{x_{k(n)}\right\}_{n \in \mathbb{N}_{0}}$ such that $\lim _{n \rightarrow \infty} x_{k(n)}=x, x \in X$. Then there is a sequence of natural numbers $\left\{p_{n}\right\}_{n \in \mathbb{N}_{0}}$ such that $x_{k(n)+p_{n}}=x_{k(n+1)}, n \in \mathbb{N}_{0}$. We have the following relations:

$$
\begin{aligned}
& \lim _{n \rightarrow \infty} x_{k(n)}=\lim _{n \rightarrow \infty} x_{k(n+1)}=\lim _{n \rightarrow \infty} x_{k(n)+p_{n}}=x, \\
& \lim _{n \rightarrow \infty} f x_{k(n)}=\lim _{n \rightarrow \infty} x_{k(n)+1}=f x, \quad \lim _{n \rightarrow \infty} f x_{k(n)+1}=f^{2} x .
\end{aligned}
$$

Suppose that $f x \neq x$. By (48), for $x=x_{k(n+1)-1}, y=x_{k(n+1)}$,

$$
\begin{aligned}
\varphi( & \left.M\left(x_{k(n+1)}, f x_{k(n+1)}, t\right)\right) \\
= & \varphi\left(M\left(x_{k(n)+p_{n}}, x_{k(n)+p_{n}+1}, t\right)\right) \\
\leq & a_{1} \varphi\left(M\left(x_{k(n)+p_{n}}, x_{k(n)+p_{n}-1}, t\right)\right) \\
& +a_{2} \varphi\left(M\left(x_{k(n)+p_{n}+1}, x_{k(n)+p_{n}}, t\right)\right)+a_{3} \varphi\left(M\left(x_{k(n)+p_{n}}, x_{k(n)+p_{n}}, t\right)\right) \\
& +a_{4} \varphi\left(M\left(x_{k(n)+p_{n}+1}, x_{k(n)+p_{n}-1}, t\right)\right) \\
& +a_{5} \varphi\left(M\left(x_{k(n)+p_{n}}, x_{k(n)+p_{n}-1}, t\right)\right), \quad n \in \mathbb{N}_{0}, t>0 .
\end{aligned}
$$

Since $(X, M, T)$ is a strong fuzzy metric space and by (47) it follows that

$$
\begin{aligned}
& \varphi\left(M\left(x_{k(n)+p_{n}+1}, x_{k(n)+p_{n}-1}, t\right)\right) \\
& \quad \leq \varphi\left(M\left(x_{k(n)+p_{n}+1}, x_{k(n)+p_{n}}, t\right)\right)+\varphi\left(M\left(x_{k(n)+p_{n}}, x_{k(n)+p_{n}-1}, t\right)\right), \quad n \in \mathbb{N}_{0}, t>0 .
\end{aligned}
$$

Now

$$
\begin{aligned}
\varphi( & \left.M\left(x_{k(n)+p_{n}}, x_{k(n)+p_{n}+1}, t\right)\right) \\
\leq & \left(a_{1}+a_{4}+a_{5}\right) \varphi\left(M\left(x_{k(n)+p_{n}}, x_{k(n)+p_{n}-1}, t\right)\right) \\
\quad & +\left(a_{2}+a_{4}\right) \varphi\left(M\left(x_{k(n)+p_{n}+1}, x_{k(n)+p_{n}}, t\right)\right), \quad n \in \mathbb{N}_{0}, t>0
\end{aligned}
$$


i.e.

$$
\begin{aligned}
\varphi & \left(M\left(x_{k(n+1)}, f x_{k(n+1)}, t\right)\right) \\
& =\varphi\left(M\left(x_{k(n)+p_{n}}, x_{k(n)+p_{n}+1}, t\right)\right) \\
& \leq \frac{a_{1}+a_{4}+a_{5}}{1-a_{2}-a_{4}} \varphi\left(M\left(x_{k(n)+p_{n}}, x_{k(n)+p_{n}-1}, t\right)\right) \\
& <\varphi\left(M\left(x_{k(n)+p_{n}}, x_{k(n)+p_{n}-1}, t\right)\right), \quad n \in \mathbb{N}_{0}, t>0 .
\end{aligned}
$$

Repeating the above procedure $p_{n}-2$ times we get

$$
\varphi\left(M\left(x_{k(n+1)}, f x_{k(n+1)}, t\right)\right)<\varphi\left(M\left(x_{k(n)+1}, f x_{k(n)+1}, t\right)\right), \quad n \in \mathbb{N}_{0}, t>0 .
$$

If we take $n \rightarrow \infty$ in (49) and apply (48) we get

$$
\begin{aligned}
\varphi(M(x, f x, t)) & \leq \varphi\left(M\left(f x, f^{2} x, t\right)\right) \\
& \leq\left(a_{1}+a_{4}+a_{5}\right) \varphi(M(x, f x, t))+\left(a_{2}+a_{4}\right) \varphi\left(M\left(f x, f^{2} x, t\right)\right)
\end{aligned}
$$

and obtain a contradiction,

$$
\varphi\left(M\left(f x, f^{2} x, t\right)\right) \leq\left(a_{1}+a_{2}+2 a_{4}+a_{5}\right) \varphi\left(M\left(f x, f^{2} x, t\right)\right)<\varphi\left(M\left(f x, f^{2} x, t\right)\right), \quad t>0 .
$$

Suppose that there exist two different fixed points $u, v \in X$. But, by (48),

$$
\varphi(M(u, v, t)) \leq\left(a_{3}+a_{4}+a_{5}\right) \varphi(M(u, v, t))<\varphi(M(u, v, t))
$$

we get a contradiction. Therefore, $x$ is a unique fixed point for $f$.

Example 2.8 Let $(X, M, T)$ be a compact strong fuzzy metric space, $X=[-2,1], T(x, y)=$ $x \cdot y, M(x, y, t)=\frac{t}{t+|x-y|}, x, y \in X, t>0$. The altering distance function is defined by $\varphi(\tau)=$ $1-\tau, \tau \in[0,1]$. We observe the continuous function

$$
f(x)= \begin{cases}-2 x, & x \in[0,1], \\ -\frac{x}{4}, & x \in[-2,0) .\end{cases}
$$

Then

$$
\varphi(M(f x, f y, t))= \begin{cases}\frac{2|x-y|}{t+2|x-y|}, & x, y \in[0,1], t>0, \\ \frac{|x-y|}{4 t+|x-y|}, & x, y \in[-2,0), t>0, \\ \frac{8 x-y}{4 t+8 x-y}, & x \in[0,1], y \in[-2,0), t>0, \\ \frac{8 y-x}{4 t+8 y-x}, & x \in[-2,0), y \in[0,1], t>0\end{cases}
$$

and

$$
\begin{aligned}
& \varphi(M(x, f x, t))=\frac{3 x}{t+3 x}, \quad x \in[0,1], t>0, \\
& \varphi(M(x, y, t))=\frac{|x-y|}{t+|x-y|}, \quad x, y \in X, t>0 .
\end{aligned}
$$


So, if we take

$$
a_{1}(x, y, t)= \begin{cases}\frac{\frac{2 x-2 y}{t+2 x-2 y}}{\frac{3 x}{t+3 x}}, & x, y \in[0,1], x>y, \\ \frac{8 x-y}{4 t+8 x-y} \\ \frac{3 x}{t+3 x} & x \in[0,1], y \in[-2,0), x \geq-\frac{y}{2},\end{cases}
$$

and $a_{2}(x, y, t)=a_{3}(x, y, t)=a_{4}(x, y, t)=a_{5}(x, y, t)=\frac{1-a_{1}(x, y, t)}{5}$ for $(x, y)$ in the aforementioned areas,

$$
a_{2}(x, y, t)= \begin{cases}\frac{\frac{2 y-2 x}{t+2 y-2 x}}{\frac{3 y}{t+3 y}}, & x, y \in[0,1], x<y, \\ \frac{8 y-x}{4 t+8 y-x}, & x \in[-2,0), y \in[0,1], y \geq-\frac{x}{2}, \\ \frac{3 y}{t+3 y} & x y-10\end{cases}
$$

and $a_{1}(x, y, t)=a_{3}(x, y, t)=a_{4}(x, y, t)=a_{5}(x, y, t)=\frac{1-a_{2}(x, y, t)}{5}$ for $(x, y)$ in the aforementioned areas,

$$
a_{5}(x, y, t)= \begin{cases}\frac{\frac{t+|x-y|}{4 t+|x-y|},}{\frac{8 x-y}{4 t+x-y}}, & x, y \in[-2,0), \\ \frac{x-y}{t+x-y}, & x \in[0,1], y \in[-2,0), x<-\frac{y}{2}, \\ \frac{8 y-x}{4 t+y-x} & x \in[-2,0), y \in[0,1], y<-\frac{x}{2},\end{cases}
$$

and $a_{1}(x, y, t)=a_{2}(x, y, t)=a_{3}(x, y, t)=a_{4}(x, y, t)=\frac{1-a_{5}(x, y, t)}{5}$ for $(x, y)$ in the aforementioned areas, condition (47) is satisfied and by Theorem 2.7 it follows that $f$ has a unique fixed point.

Note that the function $f$ does not satisfy the condition of the fuzzy Edelstein contraction theorem [29], i.e.

$$
\begin{aligned}
M(f x, f y, t) & =\frac{t}{t+2|x-y|}<\frac{t}{t+|x-y|} \\
& =M(x, y, t), \quad x, y \in[0,1], x \neq y, t>0 .
\end{aligned}
$$

Using the same altering distance, it is possible to improve the following result.

Theorem 2.9 [25] Let $(X, M, T)$ be a fuzzy metric space such that $M(x, y, t) \rightarrow 1$ as $t \rightarrow \infty$. Let $f, g: X \rightarrow X$ be two self-mappings of $X$ such that there exist $k \in(0,1)$ and $q=q(x, y, t) \geq$ 0 such that

$$
M(f x, g y, k t)+q(1-\max \{M(x, g y, k t), M(y, f x, k t)\}) \geq M(x, y, t)
$$

for each $x, y \in X$ and all $t>0$. If the family of $t$-norms $\left\{T^{(p)}(x)\right\}_{p \in \mathbb{N}}$ is equi-continuous at the point $x=1$ and $X$ is complete, then $f$ and $g$ have a common fixed point. If in (50)

$$
q(x, y, t) \leq \lambda(M(x, y, t)-M(x, y, k t)) /(1-M(x, y, k t)) \text { for all } x \neq y, \lambda \in[0,1),
$$

then the common fixed point is unique. 
Remark 2.10 A generalization of Theorem 2.9 is obtained if instead of condition (50) the following conditions hold:

$$
\begin{aligned}
& M(f x, g y, k t)+q \min \{\varphi(M(x, g y, k t)), \varphi(M(y, f x, k t))\} \\
& \quad \geq M(x, y, t), \quad x, y \in X, t>0, \\
& \lim _{n \rightarrow \infty} \mathbf{T}_{i=n}^{\infty} M\left(x_{0}, f x_{0}, \frac{1}{\mu^{i}}\right)=1
\end{aligned}
$$

for some $x_{0} \in X, \mu \in(k, 1)$ and some altering distance function $\varphi$. With $\varphi(t)=1-t$ and the $t$-norm $T$ being of $H$-type (see Proposition 1.7) we obtain a result from [25]. If we take $\varphi(t)=(1-t)^{\alpha}, \alpha>1$ we have a stronger result than in [25]. Moreover, if instead of the condition (53) the following conditions hold:

$$
\sum_{i=1}^{\infty}\left(1-M\left(x_{0}, x_{1}, \frac{1}{\mu^{i}}\right)\right)^{\lambda}<\infty
$$

and

$$
\sum_{i=1}^{\infty}\left(1-M\left(x_{0}, x_{1}, \frac{1}{\mu^{i}}\right)\right)<\infty
$$

then we obtain the same result for $T=T_{\lambda}^{\mathrm{D}}\left(T=T_{\lambda}^{\mathrm{AA}}\right), \lambda>0$, and $T=T_{\lambda}^{\mathrm{SW}}, \lambda>-1$, respectively.

The proof is similar to the one of Theorem 2.9 except for the part where it is shown that $\left\{x_{n}\right\}$ is a Cauchy sequence. In order to prove that the sequence is Cauchy let $\sigma=\frac{k}{\mu}$. Since $0<\sigma<1$ the series $\sum_{i=1}^{\infty} \sigma^{i}$ is convergent and there exists $m_{0} \in \mathbb{N}$ such that $\sum_{i=m_{0}}^{\infty} \sigma^{i}<1$. Hence for every $m>m_{0}$ and every $s \in \mathbb{N}$,

$$
t>t \sum_{i=m_{0}}^{\infty} \sigma^{i}>t \sum_{i=m}^{m+s-1} \sigma^{i}
$$

which implies that

$$
\begin{aligned}
M\left(x_{m+s}, x_{m}, t\right) \geq & M\left(x_{m+s}, x_{m}, t \sum_{i=m}^{m+s-1} \sigma^{i}\right) \\
\geq & \underbrace{T(T(\cdots T}_{s \text {-times }}\left(M\left(x_{m+s}, x_{m+s-1}, t \sigma^{m+s-1}\right), M\left(x_{m+s-1}, x_{m+s-2}, t \sigma^{m+s-2}\right), \ldots,\right. \\
& \left.\left.\left.M\left(x_{m+1}, x_{m}, t \sigma^{m}\right)\right)\right)\right) \\
\geq & \underbrace{T(T(\cdots T}_{s-\text { times }}\left(M\left(x_{0}, x_{1}, \frac{t \sigma^{m+s-1}}{k^{m+s-1}}\right), M\left(x_{0}, x_{1}, \frac{t \sigma^{m+s-2}}{k^{m+s-2}}\right), \ldots,\right. \\
& \left.\left.\left.M\left(x_{0}, x_{1}, \frac{t \sigma^{m-1}}{k^{m-1}}\right)\right)\right)\right) \\
\geq & \mathbf{T}_{i=m}^{m+s-1} M\left(x_{0}, x_{1}, \frac{t}{\mu^{i}}\right) \geq \mathbf{T}_{i=m}^{\infty} M\left(x_{0}, x_{1}, \frac{t}{\mu^{i}}\right), \quad t>0 .
\end{aligned}
$$


By Theorem 1.8, $\lim _{n \rightarrow \infty} \mathbf{T}_{i=m}^{\infty} M\left(x_{0}, x_{1}, \frac{1}{\mu^{i}}\right)=1$ implies that $\lim _{n \rightarrow \infty} \mathbf{T}_{i=m}^{\infty} M\left(x_{0}, x_{1}, \frac{t}{\mu^{i}}\right)=1$, $t>0$. Hence, for every $\varepsilon \in(0,1)$ there exists $m_{1} \in \mathbb{N}$ such that

$$
M\left(x_{m+s}, x_{m}, t\right)>1-\varepsilon, \quad m \geq m_{1}, s \in \mathbb{N}, t>0,
$$

i.e. $\left\{x_{n}\right\}_{n \in \mathbb{N}}$ is a Cauchy sequence.

Theorem 2.11 Let $(X, M, T)$ and $(X, \tilde{M}, T)$ be fuzzy metric spaces such that $\lim _{t \rightarrow \infty} \tilde{M}(x$, $y, t)=1$ and $M(x, y, t) \geq \tilde{M}(x, y, t), x, y \in X, t>0$. Let $f: X \rightarrow X$ be a continuous function such that for some $x_{0} \in X$ the sequence $\left\{f^{n} x_{0}\right\}$ has a convergent subsequence in $(X, M, T)$. If there exist $k \in(0,1), \mu \in(k, 1), q=q(x, y, t) \geq 0$, and an altering distance function $\varphi$ such that the following conditions are satisfied:

$$
\begin{gathered}
\tilde{M}(f x, f y, k t)+q \min \{\varphi(\tilde{M}(f x, y, k t)), \varphi(\tilde{M}(f y, x, k t))\} \\
\geq \tilde{M}(x, y, t), \quad x, y \in X, t>0
\end{gathered}
$$

and

$$
\lim _{n \rightarrow \infty} \mathbf{T}_{i=n}^{\infty} \tilde{M}\left(x_{0}, f x_{0}, \frac{1}{\mu^{i}}\right)=1
$$

then $f$ has a fixed point. If, in addition, the following is satisfied:

$$
q(x, y, t) \leq \lambda(\widetilde{M}(x, y, t)-\tilde{M}(x, y, k t)) / \varphi(\tilde{M}(x, y, k t)), \quad x, y \in X, x \neq y, \lambda \in[0,1),
$$

then $f$ has a unique fixed point.

Proof We define the sequence $x_{n}=f x_{n-1}=f^{n} x_{0}, n \in \mathbb{N}$. By (56) with $x=x_{n-1}$ and $y=x_{n}$ we get

$$
\widetilde{M}\left(x_{n}, x_{n+1}, t\right) \geq \tilde{M}\left(x_{n-1}, x_{n}, t\right) \geq \cdots \geq \tilde{M}\left(x_{0}, x_{1}, \frac{t}{k^{n}}\right), \quad n \in \mathbb{N}, t>0 .
$$

The proof that $\left\{x_{n}\right\}$ is a Cauchy sequence in $(X, \tilde{M}, T)$ is the same as in Remark 2.10. Then $\left\{x_{n}\right\}$ is a Cauchy sequence in $(X, M, T)$, too. Since, by assumption, the Cauchy sequence $\left\{x_{n}\right\}$ has a convergent subsequence in $(X, M, T)$, there exists $p \in X$ such that $\lim _{n \rightarrow \infty} x_{n}=p$.

Since the function $f$ is continuous we have

$$
f p=f \lim _{n \rightarrow \infty} x_{n}=\lim _{n \rightarrow \infty} f x_{n}=\lim _{n \rightarrow \infty} x_{n+1}=p
$$

and $f$ has a fixed point. Let us prove that the fixed point is unique. Suppose that $p$ and $w$ are two different fixed points. Then, by (56) with $x=p$ and $y=w$, we have

$$
\tilde{M}(f p, f w, k t)+q \min \{\varphi(\tilde{M}(f p, w, k t)), \varphi(\tilde{M}(f w, p, k t))\} \geq \tilde{M}(p, w, t), \quad t>0
$$

Using (57), it follows that $\tilde{M}(p, w, k t) \geq \tilde{M}(p, w, t), t>0$, i.e. $p=w$. So, the fixed point is unique. 


\section{Conclusion}

In this paper several fixed point theorems in complete and compact fuzzy metric spaces are proved. For this purpose new contraction types of mappings with altering distances are proposed. The theorems presented with appropriate examples improve some recently published results.

The following question can be asked:

Is it possible to omit the assumption that the t-norm is non-nilpotent in Theorem 2.5?

\section{Competing interests}

The authors declare that they have no competing interests.

\section{Authors' contributions}

All authors contributed equally to the writing of this paper. All authors read and approved the final manuscript.

\section{Author details}

${ }^{1}$ Faculty of Science and Technology, Valaya Alongkorn Rajabhat University under the Royal Patronage, 1 Moo 20 Phaholyothin Rd., Klong Neung, Klong Luang, Pathumthani, 13180, Thailand. ${ }^{2}$ Faculty of Technology, University of Novi Sad, Novi Sad, Serbia. ${ }^{3}$ Department of Applied Mathematics and Humanities, s.v. National Institute of Technology, Surat, Gujarat 395007, India. ${ }^{4}$ Department of Mathematics, Faculty of Science, King Mongkut's University of Technology Thonburi (KMUTT), 126 Pracha Uthit Rd., Bang Mod, Thung Khru, Bangkok, 10140, Thailand.

\section{Acknowledgements}

The authors thank the editor-in-chief and the referee(s) for their valuable comments and suggestions, which were very useful in improving the presentation of the paper. Tatjana Došenović, Dušan Rakić wish to thank the projects MNTRRS-174009, III44006, and PSNTR Project No. 114-451-2167 that enabled this research, and Dhananjay Gopal is grateful for the support of CSIR, Govt. of India, Grant No.-25(0215)/13/EMR-II. Moreover, Poom Kumam was supported by the Higher Education Research Promotion and National Research University Project of Thailand, Office of the Higher Education Commission (NRU2558).

Received: 20 December 2014 Accepted: 17 April 2015 Published online: 14 May 2015

\section{References}

1. Banach, S: Sur les opérations dans les ensembles abstraits et leur application aux équations intégrales. Fundam. Math. 3, 133-181 (1922)

2. George, A, Veeramani, P: On some results in fuzzy metric spaces. Fuzzy Sets Syst. 64, 395-399 (1994)

3. George, A, Veeramani, P: On some results of analysis for fuzzy metric spaces. Fuzzy Sets Syst. 90, 365-368 (1997)

4. Kramosil, I, Michalek, J: Fuzzy metric and statistical metric spaces. Kybernetika 11, 336-344 (1975)

5. Zadeh, LA: Fuzzy sets. Inf. Control 8, 338-353 (1965)

6. Altun, I, Mihet, D: Ordered non-Archimedean fuzzy metric spaces and some fixed point results. Fixed Point Theory Appl. 2010, 782680 (2010)

7. Choudhury, BS, Das, K, Dutta, PN: A fixed point result in Menger spaces using a real function. Acta Math. Hung. 122, 203-216 (2009)

8. Hadžić, O: A fixed point theorem in Menger spaces. Publ. Inst. Math. (Belgr.) 20, 107-112 (1979)

9. Hadžić, O: Fixed Point Theory in Probabilistic Metric Spaces. Serbian Academy of Sciences and Arts, Branch in Novi Sad, University of Novi Sad, Institute of Mathematics, Novi Sad (1995)

10. Hadžić, O, Pap, E: Fixed Point Theory in Probabilistic Metric Spaces. Kluwer Academic, Dordrecht (2001)

11. Hong, S: Fixed points for modified fuzzy $\psi$-contractive set-valued mappings in fuzzy metric spaces. Fixed Point Theory Appl. 2014, 12 (2014)

12. Mihet, D: A Banach contraction theorem in fuzzy metric spaces. Fuzzy Sets Syst. 144, 431-439 (2004)

13. Saadati, R, Kumam, P, Jang, S: On the tripled fixed point and tripled coincidence point theorems in fuzzy normed spaces. Fixed Point Theory Appl. 2014, 136 (2014)

14. Shen, $Y$, Chen, W, Wang, S: Comment on 'Common fixed point theorems for commutating mappings in fuzzy metric spaces'. Abstr. Appl. Anal. (2012). doi:10.1155/2012/142858

15. Wang, S, Alsulami, SM, Ćirić, L: Common fixed point theorems for nonlinear contractive mappings in fuzzy metric spaces. Fixed Point Theory Appl. 2013, 191 (2013)

16. Fuller, R: Neural fuzzy systems. Abo Akademis tryckeri, Abo, ESF Series A:443 (1995)

17. McBratney, A, Odeh, IOA: Application of fuzzy sets in soil science: fuzzy logic, fuzzy measurements and fuzzy decisions. Geoderma 77, 85-113 (1997)

18. El Naschie, MS: The two-slit experiment as the foundation of $E$-infinity of high energy physics. Chaos Solitons Fractals 25, 509-514 (2005)

19. Romaguera, S, Sapena, A, Tirado, P: The Banach fixed point theorem in fuzzy quasi-metric spaces with application to the domain of words. Topol. Appl. 154, 2196-2203 (2007)

20. Steimann, F: On the use and usefulness of fuzzy sets in medical Al. Artif. Intell. Med. 21, 131-137 (2001)

21. Khan, MS, Swaleh, M, Sessa, S: Fixed point theorems by altering distances between the points. Bull. Aust. Math. Soc. 30, 1-9 (1984)

22. Choudhury, BS, Das, K: A new contraction principle in Menger spaces. Acta Math. Sin. 24, 1379-1386 (2008)

23. Mihet, D: Altering distances in probabilistic Menger spaces. Nonlinear Anal. 71, 2734-2738 (2009) 
24. Shen, Y, Qiu, D, Chen, W: Fixed point theorems in fuzzy metric spaces. Appl. Math. Lett. 25, 138-141 (2012)

25. Ćirić, L: Some new results for Banach contractions and Edelstein contractive mappings on fuzzy metric spaces. Chaos Solitons Fractals 42, 146-154 (2009)

26. Klement, EP, Mesiar, R, Pap, E: Triangular Norms. Trends in Logic, vol. 8. Kluwer Academic, Dordrecht (2000)

27. Gregori, V, Morillas, S, Sapena, A: On a class of compatible fuzzy metric spaces. Fuzzy Sets Syst. 161, 2193-2205 (2010)

28. Rodriguez-Lopez, J, Romaguera, S: The Hausdorff fuzzy metric on compact sets. Fuzzy Sets Syst. 147, $273-283$ (2004)

29. Grabiec, M: Fixed points in fuzzy metric spaces. Fuzzy Sets Syst. 27, 385-389 (1988)

30. Hadžić, O, Pap, E, Budinčević, M: Countable extension of triangular norms and their applications to the fixed point theory in probabilistic metric spaces. Uncertainty modelling, 2001 (Bratislava). Kybernetika 38(3), 363-382 (2002)

31. Žikić, T: On fixed point theorems of Gregori and Sapena. Fuzzy Sets Syst. 144, 421-429 (2004)

32. Wardowski, D: Fuzzy contractive mappings and fixed points in fuzzy metric space. Fuzzy Sets Syst. 222, 108-114 (2013)

\section{Submit your manuscript to a SpringerOpen ${ }^{\circ}$ journal and benefit from:}

- Convenient online submission

Rigorous peer review

- Immediate publication on acceptance

- Open access: articles freely available online

- High visibility within the field

- Retaining the copyright to your article 